\title{
El Practicum en Psicopedagogía: análisis comparativo entre áreas de conocimiento
}

\section{Practicum in Educational Psychology: comparative analysis between areas of knowledge}

\author{
Eva Ma ESPIÑEIRA BELLÓN, Alicia ARIAS RODRÍGUEZ y \\ Nuria REBOLLO QUINTELA \\ Universidad de A Coruña
}

Recibido: Julio 2014

Evaluado: Noviembre 2014

Aceptado: Diciembre 2014

\begin{abstract}
Resumen
En el presente artículo presentamos el proceso y los resultados de una investigación en la que, tras reflexionar sobre el concepto de Practicum y, de manera específica, sobre el Practicum de la titulación de Psicopedagogía impartida en la Universidad de A Coruña, se realiza una evaluación teniendo en cuenta la opinión del alumnado que ha cursado dicha materia, en cuanto a la relevancia que le otorga a los objetivos planificados y la posterior consecución de éstos. Los resultados se diferencian teniendo en cuenta el área a la que pertenece el profesorado que lo ha tutorizado. En lo metodológico, es una investigación descriptiva, tipo encuesta, desde la que se interviene en una muestra significativa del alumnado de último curso de la titulación en el año 2012/13 a través de un cuestionario, cuya validez (didáctica y contenido) y fiabilidad (consistencia interna) han sido debidamente establecidas (juicio de expertos y una experiencia piloto para el cálculo del alfa de Cronbach). Los resultados muestran que el programa de Practicum presenta un buen desarrollo de la mayoría de los objetivos, sobre todo los referidos a habilidades, en donde las medias de los objetivos mejor valorados superan la puntuación de 4 . Esto último pone de manifiesto claras líneas de innovación y de mejora en los ámbitos a nivel conceptual y actitudinal.
\end{abstract}

Palabras clave: practicum, psicopedagogía, áreas de conocimiento, habilidades.

\begin{abstract}
In this paper we present the process and results of an investigation in which, after reflecting on the concept of Practicum and, specifically, in the Practicum in Educational Psychology degree taught at the University of A Coruña, an assessment is made taking into account the opinion of the students who have studied this matter, as to the relevance that the planned objectives are given and subsequent achievement of these. The results differ considering the area that the teacher who has tutored belongs to. Methodologicaly, it is a descriptive research, survey type, which involved a significant sample of students in their final year of the degree in the year 2012/13 through a questionnaire which validity (Teaching and content) and reliability (internal consistency) have been properly set (judgment of experts and a pilot experience for the calculation of Cronbach's alpha). The results show that the Practicum program has a good


development of most of the objectives, especially those related to skills, where the average of the highest rated goals exceed the rating of 4 . This suggests clear lines for innovation and improvement in the areas of conceptual and attitudinal level.

Keywords: practicum, psychology, areas of knowledge, skill.

Entenderemos por prácticas en empresas, al igual que lo hace Cunill Mir (2009, p. 408) como "período de estancia de un alumno en una empresa, institución o entidad que tiene por objetivo aplicar los conocimientos teóricos adquiridos a lo largo de su carrera, el adquirir destrezas y habilidades para su desarrollo profesional, y el de ser capaz de establecer vínculos sociales con el resto de los trabajadores”. Hemos elegido esta definición ya que señala los objetivos generales que luego se concretan en objetivos más específicos, objetivos relacionados con los del Practicum en Psicopedagogía, diferenciados en conceptuales, de habilidades y destrezas y a nivel de actitudes.

En la publicación del Plan de Estudios de la Licenciatura de Psicopedagogía (BOE de 16 de agosto de 1993) el Practicum se define como el "conjunto integrado de prácticas que proporcionan experiencia directa sobre diversos aspectos de la intervención psicopedagógica” y se establece como una materia de segundo curso de la titulación. La consideración como materia troncal y el número de créditos dedicados (12c), parecen reforzar su importancia de cara a la formación de las futuras y futuros psicopedagogas/os.

Algo a destacar del Plan de Estudios es que es una materia adscrita a las siguientes áreas de conocimiento: Didáctica y Organización Escolar (DOE), Métodos de Investigación y Diagnóstico en Educación (MIDE), Psicología Evolutiva y de la Educación (PEE) y Teoría e Historia de la Educación (THE). El profesorado de cada una de las áreas anteriores se encarga de tutorizar al grupo de alumnas/os. De esta forma, el Practicum de Psicopedagogía, constituye para el alumnado, como señala Domingo Roget (2009) "una fuente de nuevos y variados aprendizajes, estrechamente relacionados con las disciplinas estudiadas en la institución universitaria” (p.125).

El Practicum ha ocupado un lugar destacado en aquellas titulaciones en las que se reconoce la necesidad de ofrecer una formación en la que se combinen los componentes teóricos y prácticos. De hecho, existen numerosas investigaciones en las que la materia de Practicum es objeto de estudio en diferentes titulaciones relacionadas con las Ciencias de la Educación (educación social, logopedia, magisterio, máster de profesorado de educación secundaria, pedagogía o psicopedagogía) (Armengol, Castro, Jariot, Massot y Sala, 2011; Bretones Román, 2013; Cabrerizo Diago, Rubio Roldán y Castillo Arredondo, 2010; Cabrerizo Diago, Rubio Roldán, Castillo Arredondo y Cabrerizo García, 2011; González Fernández, 2006; Latorre Medina y Blanco Encomienda, 2009; Rodicio e Iglesias, 2011).

También hoy en día, es objeto de debate en el proceso de implantación del Espacio Europeo de Educación Superior, de tal manera que, como señalan Armengol, Castro, Jariot, Massot y Sala (2011) “se aproxime al máximo a una situación profesional real” (p.71), cuestionándose aspectos relacionados con las funciones que puedan desarrollar los/las profesionales, los ámbitos de trabajo más frecuentes y, a diferencia de los 
estudios planificados con anterioridad, centrados en objetivos, las competencias que el alumnado ha de adquirir en dichos contextos.

Otras temáticas se centran en el análisis de la acción tutorial que desempeña el profesorado tutor del Practicum o en comprobar la incidencia de dicho Practicum en la formación teórico-práctica del/de la futuro/a psicopedagogo/a. También existen estudios que priorizan la importancia del papel del profesorado tutor de las entidades colaboradoras. En cuanto a la titulación de psicopedagogía en concreto, también se han analizado diversos datos que han llevado a autores y autoras a establecer numerosos estudios teóricos a nivel de objetivos y competencias a alcanzar por el alumnado de psicopedagogía (Armengol, Castro, Jariot, Massot y Sala, 2011; Cid y Ocampo, 2007; Cubo y Montanero, 2000; Latorre y Blanco, 2009; Martínez Figueira, 2005, 2008; Rodicio e Iglesias, 2011; Tello, 2007; Vallejos, Fernández y Sánchez (2002).

En este caso, se ha optado por un aspecto no tratado en las investigaciones consultadas, sino más relacionado con el efectuado por Tello (2007) en el que se hace referencia a las asignaturas de mayor y menor utilidad o aplicabilidad para el desarrollo del Practicum. Concordamos con Martín Pérez (2008) cuando señala que el profesorado tutor de la universidad "es igualmente, una pieza clave para que el alumnado logre extraer conocimientos de las experiencias en los centros de trabajo" (p.24). En este sentido, en el presente trabajo intentaremos extraer las principales conclusiones referentes a los resultados extraídos en función del área de conocimiento del profesorado tutor que tutoriza al alumnado de Practicum desde la universidad.

\section{Método}

\section{Objetivos}

Basándonos en los objetivos de la titulación y teniendo en cuenta que el Practicum es el lugar y el momento idóneo para la comprobación de la consecución de los objetivos de las diferentes materias por el alumnado de Psicopedagogía decidimos poner en marcha una pequeña investigación con los siguientes objetivos:

- Caracterizar la muestra en función de las variables de identificación.

- Determinar si existen diferencias, entre las valoraciones realizadas por el alumnado sobre su percepción de los objetivos en función de las tres áreas de conocimiento que han tutorizado el Practicum (DOE, MIDE y PEE), atendiendo, por un lado a la consecución de éstos y, por el otro, a la importancia que nuestro alumnado les otorga.

\section{Muestra}

En relación con la muestra, el Practicum constituye, como señala Zabalza (2013), "una realidad compleja, determinada por múltiples variables y en la que participan tres agentes fundamentales: los estudiantes, las instituciones universitarias y los centros de prácticas” (p. 21). 
Esta investigación, de carácter exploratorio y piloto, cuenta en el curso 2012/2013, con una población de 51 estudiantes matriculados/as en la materia de Practicum en la titulación de Psicopedagogía de la Facultad de Ciencias de la Educación de la Universidad de A Coruña.

La muestra está compuesta por un total de 31 sujetos. El error aleatorio de muestreo, a partir del supuesto de máxima indeterminación $(\mathrm{p}=\mathrm{q}=.50)$ y con un margen de confianza del $95.5 \%$, fue de $11,3 \%$. El $87 \%$ son mujeres; no hay que olvidar que estamos en una titulación mayoritariamente feminizada, y el $13 \%$ son hombres. El muestreo es intencional, ya que, se ha encuestado al alumnado asistente al aula de una materia del segundo cuatrimestre de dicha titulación, que había cursado durante el año académico 2012-2013 la materia de Practicum.

Con respecto a la tutorización de este alumnado en dicha materia, de los 31 sujetos encuestados, un 51,6\% fue tutorizado por profesorado del área de Didáctica y Organización Escolar (DOE); un 35,5\% por profesorado del área de MIDE y un 12.9\% del área de Psicología Evolutiva y de la Educación (PEE).

En el proceso previo de decisión del centro donde el alumnado realizará el Practicum, entran en consideración, como señala Castilla Mesa (2009), intereses y motivaciones personales, condicionantes provenientes de los contextos profesionales donde realizará esta asignatura y factores organizativos y estructurales del Practicum, estrictamente académicos. "El proceso decisorio es complejo y quizá sea éste uno de los momentos que puede generar más ansiedad y expectativas dentro de la trayectoria formativa académica” (p. 355).

El ámbito de intervención, en el que este alumnado ha realizado el Practicum destaca con un $38,7 \%$ en la Unidad o Departamento de Orientación en un centro educativo; un 38,7\% en la categoría "Otras" que recoge ámbitos como la Asociación familiar y de enfermos de Alzheimer; la Unidad Municipal de Atención a Drogodependientes (UMAD); la Asociación Stop accidentes; la Asociación Ciudadana de Lucha Ante la Droga (ACLAD); el Complejo Hospitalario Universitario de A Coruña (CHUAC); el Centro de Formación y Recursos (CEFORE) y el Centro de atención de adultos con discapacidad psíquica. También, los/las estudiantes realizan las prácticas en los Equipos Psicopedagógicos de Apoyo (9,7\%) y en los Gabinetes psicopedagógicos de ejercicio privado (9,7\%).

En cuanto a la elección del ámbito de intervención por parte del alumnado, un $61,3 \%$ ha elegido por petición propia; un $29 \%$ a través del listado facilitado por la facultad y un $9,7 \%$ por sorteo.

\section{Procedimiento}

Visto el desarrollo del primero de los objetivos de la investigación, el segundo se centró en investigar si, con respecto a los objetivos especificados en el Practicum de la titulación, existen diferencias, entre las valoraciones realizadas por el alumnado de acuerdo a las dos dimensiones presentadas (consecución y relevancia), teniendo en 
cuenta para ello, las diferentes áreas de conocimiento (Espiñeira, Muñoz, Rebollo y Arias, 2013).

Objetivos de carácter específico:

A nivel conceptual:

- Integrar los conocimientos teórico-prácticos en la identificación y solución de los problemas que competen a su campo profesional.

- Identificar en el medio social real problemas que se presentan en la comunidad escolar y aquellos programas encaminados a su solución y prevención.

- Identificar y describir las funciones del/de la psicopedagogo/a en su desempeño profesional y evaluar críticamente sus limitaciones.

A nivel de habilidades y destrezas:

- Colaborar en la planificación y en el desarrollo de las actividades pedagógicas.

- Colaborar con personal especializado en la evaluación de los problemas específicos de la evaluación e intervención psicopedagógica.

- Diseñar, aplicar y evaluar programas educativos a nivel grupal y/o individual.

- Conocer y adaptar los recursos disponibles en el medio institucional para conseguir sus objetivos profesionales.

- Diseñar, aplicar y evaluar materiales y recursos necesarios para el óptimo desarrollo de su actividad profesional.

- Colaborar con otros profesionales en los procesos educativos que se desarrollan en las distintas instituciones implicadas en la intervención psicopedagógica.

- Diseñar, aplicar y evaluar instrumentos de recogida de datos.

- Sistematizar, describir e interpretar los datos recogidos.

A nivel de actitudes:

- Demostrar una actitud positiva cara a la investigación de los procesos involucrados en su práctica profesional, la comprobación de la efectividad de sus técnicas y la búsqueda de alternativas prácticas que mejoren la calidad de su trabajo profesional.

- Mostrar interés cara a la actualización permanente a través de la lectura crítica de estudios, investigaciones y reflexiones relacionados con su área de trabajo.

Tabla 1. Objetivos de la materia de Practicum de la titulación de Psicopedagogía de la Facultad de Ciencias de la Educación de la Universidad de A Coruña

La actividad realizada durante el Practicum, implica, como señalan Cabrerizo Diago, Rubio Roldán y Castillo Arredondo (2010), contar con un conocimiento teórico inicial, de ahí el desarrollo de objetivos a nivel conceptual que permitan abordar y adquirir la formación práctica (objetivos referidos a habilidades y destrezas) y reflexionar sobre el sentido que este conocimiento práctico proporciona (objetivos a nivel de actitudes). Se pretende que el alumnado de Practicum "analice, contextualice y reflexione acerca de las estrategias, las técnicas e instrumentos y las actuaciones a desarrollar en cada caso, en un proceso de indagación constante sobre la práctica profesional” (p. 121). En este sentido, hemos preparado el cuestionario con el objetivo de recoger la información que el alumnado señala en función de las diferentes áreas de conocimiento.

Los objetivos específicos que se pretenden alcanzar mediante la realización del Practicum en la titulación de Psicopedagogía de la Facultad de Ciencias de la Educación de la Universidad de A Coruña se concretan atendiendo a los tres ámbitos 
de conocimiento a nivel conceptual, de habilidades y destrezas y a nivel de actitudes, como figuran en la Tabla 1.

\section{Instrumento}

Inicialmente se construyó un cuestionario tipo Likert con cinco opciones de respuesta: Ninguna (1); Poca (2); Regular (3); Bastante (4) y Mucha (5), en el que se recogían datos de las variables independientes, para caracterizar la muestra, como por ejemplo, sexo, área de conocimiento de su tutor/a de Practicum en la facultad, ámbito de intervención en que realiza las prácticas y razón de elección del centro en el que se realizan las prácticas. Y como variables dependientes, se han diseñado dos grandes dimensiones: consecución y relevancia.

En segundo lugar, se determinó la validez de constructo mediante la técnica del juicio de expertos, seleccionando cinco especialistas versados en la temática, quienes analizaron cada uno de los ítems en cuanto a la claridad, congruencia y tendenciosidad. Posteriormente, se realizaron las modificaciones sugeridas por los/las expertos/as, dando como resultado un instrumento depurado de 26 ítems.

De esta forma, se redactó un pequeño cuestionario en el que el alumnado fuese capaz de detectar cuáles de los objetivos planificados eran capaces de alcanzar y a cuáles les otorgaban una mayor/menor importancia. No se incorpora dicho cuestionario, por cuestiones de espacio, teniendo en cuenta que los diferentes ítems se pueden consultar en las Tablas 2 - 7.

\section{Resultados: análisis descriptivo}

Se realiza, a continuación, un análisis respecto a los tres tipos de objetivos específicos (a nivel conceptual, de habilidades y de actitudes) más y menos valorados por el alumnado con respecto a las dos dimensiones estudiadas (consecuencia y relevancia) teniendo en cuenta el alumnado tutorizado por profesorado de las diferentes áreas de conocimiento: MIDE, DOE y PEE, debido a que en este curso ningún/a alumno/a ha sido tutorizado por profesorado del Área de Teoría e Historia de la Educación (THE). (Ver tablas 2, 3 y 4).

En cuanto a la dimensión relacionada con la consecución de los objetivos a nivel conceptual, como se puede observar en la tabla 2, los objetivos que alcanzan medias más altas por el alumnado tutorizado por el profesorado del área DOE son "pudiste identificar problemas, aplicando los conocimientos teórico-prácticos” y "pudiste identificar las funciones de la/el psicopedagoga/o”, ambos con una media de 3.69 y una desviación típica de 1.01 el primero y un 1.40 el segundo.

Estos mismos objetivos alcanzan medias altas para el alumnado tutorizado por profesorado del área MIDE. Pero con la salvedad de que el objetivo con una media mayor por este alumnado es el objetivo "pudiste identificar las funciones de la/el psicopedagoga/o" con una media de 3.45 y una desviación típica de 1.57. 
En cambio, para el alumnado que es tutorizado por el profesorado del área PEE el objetivo que obtiene mayor media es "pudiste identificar programas encaminados a la solución y/o prevención de problemas” (media 2.25 y desviación típica .95).

\begin{tabular}{|c|c|c|c|}
\hline \multirow{2}{*}{$\begin{array}{c}\text { Medias y desviaciones típicas de los objetivos conceptuales en la dimensión } \\
\text { consecución }\end{array}$} & \multicolumn{3}{|c|}{ Consecución } \\
\hline & Área & Media & DT \\
\hline \multirow[t]{3}{*}{ Pudiste identificar problemas, aplicando los conocimientos teórico-prácticos } & $\mathrm{DOE}$ & 3.69 & 1.014 \\
\hline & MIDE & 2.82 & 1.168 \\
\hline & PEE & 2.00 & 0.816 \\
\hline \multirow[t]{3}{*}{ Pudiste resolver problemas, aplicando los conocimientos teórico-prácticos } & $\mathrm{DOE}$ & 3.06 & 1.124 \\
\hline & MIDE & 2.55 & 1.128 \\
\hline & PEE & 2.00 & 0.816 \\
\hline \multirow{3}{*}{$\begin{array}{l}\text { Pudiste identificar programas encaminados a la solución y/o prevención de } \\
\text { problemas }\end{array}$} & $\mathrm{DOE}$ & 3.00 & 1.265 \\
\hline & MIDE & 2.55 & 1.28 \\
\hline & PEE & 2.25 & 0.957 \\
\hline \multirow[t]{3}{*}{ Pudiste identificar las funciones de la/el psicopedagoga/o } & DOE & 3.69 & 1.401 \\
\hline & MIDE & 3.45 & 1.572 \\
\hline & PEE & 1.50 & 0.577 \\
\hline \multirow{3}{*}{$\begin{array}{l}\text { Pudiste evaluar críticamente las limitaciones de las funciones de la/el } \\
\text { psicopedagoga/o }\end{array}$} & DOE & 3.19 & 1.109 \\
\hline & MIDE & 2.91 & 1.375 \\
\hline & PEE & 1.75 & 0.957 \\
\hline
\end{tabular}

Tabla 2. Medias y desviaciones típicas de los objetivos conceptuales en la dimensión consecución

En la misma tabla 2, destaca como el objetivo que obtiene medias más bajas, para el alumnado tutorizado por el profesorado del área DOE es "pudiste identificar programas encaminados a la solución y/o prevención de problemas” con una media 3 y una desviación típica .126.

Este mismo objetivo es, también, para el alumnado que tutoriza el profesorado de MIDE el que posee una media más baja de 2.55 y una desviación típica de 1,28. Para este último grupo de alumnos/as existe otro objetivo "pudiste, resolver problemas, aplicando los conocimientos teóricos-prácticos” con una media de 2.55 y una desviación típica de 1.12 .

Por último, cabe destacar que el objetivo menos valorado por el alumnado tutorizado por el profesorado de PEE es "pudiste identificar las funciones de la/el psicopedagoga/o” con una media de 1.50 y una desviación típica de .57.

En la dimensión relacionada con la consecución de los objetivos a nivel de habilidades, los objetivos con medias más altas para el alumnado tutorizado por el profesorado del área DOE son "pudiste conocer recursos disponibles en el medio institucional" con una media de 4.31 y una desviación típica de .79 y "pudiste colaborar en el desarrollo de actividades pedagógicas" con una media de 4.19 y una desviación típica de 1.04 . 
Estos mismos objetivos, también, obtienen medias más altas para el alumnado tutorizado por el profesorado del área MIDE ambos, con una media de 4.09 y 1.22 respectivamente.

Para el alumnado tutorizado por el profesorado del área PEE el único objetivo con una media de 4 y una desviación típica de 1.15 es "pudiste conocer recursos disponibles en el medio institucional” (ver tabla 3).

\begin{tabular}{|c|c|c|c|}
\hline \multirow{2}{*}{$\begin{array}{c}\text { Medias y desviaciones típicas de los objetivos referidos a habilidades } \\
\text { en la dimensión consecución }\end{array}$} & \multicolumn{3}{|c|}{ Consecución } \\
\hline & Área & Media & DT \\
\hline \multirow[t]{3}{*}{ Pudiste colaborar en la planificación de actividades pedagógicas } & DOE & 3.94 & 0.680 \\
\hline & MIDE & 3.45 & 1.508 \\
\hline & PEE & 2.50 & 1.000 \\
\hline \multirow[t]{3}{*}{ Pudiste colaborar en el desarrollo de actividades pedagógicas } & DOE & 4.19 & 1.047 \\
\hline & MIDE & 4.09 & 1.221 \\
\hline & PEE & 3.75 & 1.893 \\
\hline \multirow{3}{*}{$\begin{array}{l}\text { Pudiste colaborar con personal especializado en la evaluación e } \\
\text { intervención psicopedagógica }\end{array}$} & DOE & 3.31 & 1.448 \\
\hline & MIDE & 3.00 & 1.673 \\
\hline & PEE & 2.000 & 1.000 \\
\hline \multirow[t]{3}{*}{ Pudiste colaborar con otras/os profesionales en los procesos educativos } & DOE & 3.44 & 1.365 \\
\hline & MIDE & 3.73 & 1.489 \\
\hline & PEE & 2.000 & 0.816 \\
\hline \multirow[t]{3}{*}{ Pudiste conocer recursos disponibles en el medio institucional } & DOE & 4.31 & 0.793 \\
\hline & MIDE & 4.09 & 1.221 \\
\hline & PEE & 4.00 & 1.155 \\
\hline \multirow[t]{3}{*}{ Pudiste adaptar recursos disponibles en el medio institucional } & DOE & 3.44 & .814 \\
\hline & MIDE & 3.64 & 1.362 \\
\hline & PEE & 3.25 & 0.500 \\
\hline \multirow[t]{3}{*}{ Pudiste diseñar programas educativos } & DOE & 2.25 & 1.483 \\
\hline & MIDE & 2.18 & 1.079 \\
\hline & PEE & 1.25 & 0.500 \\
\hline \multirow[t]{3}{*}{ Pudiste diseñar materiales y recursos necesarios } & DOE & 2.94 & 1.482 \\
\hline & MIDE & 2.64 & 1.629 \\
\hline & PEE & 3.75 & 0.500 \\
\hline \multirow[t]{3}{*}{ Pudiste diseñar instrumentos de recogida de datos } & DOE & 2.44 & 1.672 \\
\hline & MIDE & 1.82 & 1.079 \\
\hline & PEE & 1.75 & 0.957 \\
\hline \multirow[t]{3}{*}{ Pudiste aplicar programas educativos } & DOE & 2.65 & 1.711 \\
\hline & MIDE & 2.64 & 1.567 \\
\hline & PEE & 1.75 & 0.500 \\
\hline \multirow[t]{3}{*}{ Pudiste aplicar materiales y recursos } & $\mathrm{DOE}$ & 3.75 & 1.342 \\
\hline & MIDE & 3.45 & 1.368 \\
\hline & PEE & 3.67 & 1.155 \\
\hline
\end{tabular}

Tabla 3. Medias y desviaciones típicas de los objetivos referidos a habilidades en la dimensión consecución 


\begin{tabular}{llll} 
Medias y desviaciones típicas de los objetivos referidos a habilidades & \multicolumn{3}{c}{ Consecución } \\
en la dimensión consecución & \multicolumn{1}{c}{ Área } & Media & \multicolumn{1}{c}{ DT } \\
\hline Pudiste aplicar instrumentos de recogida de datos & DOE & 3.56 & 1.590 \\
\cline { 2 - 4 } & MIDE & 2.36 & 1.502 \\
\hline Pudiste sistematizar los datos recogidos (si los aplicaste) & PEE & 1.75 & 0.957 \\
\cline { 2 - 4 } & DOE & 3.33 & 1.759 \\
\hline Pudiste describir los datos recogidos (si los aplicaste) & PEE & 1.75 & 0.957 \\
\hline Pudiste interpretar los datos recogidos (si los aplicaste) & DOE & 3.47 & 1.552 \\
\cline { 2 - 4 } & MIDE & 1.90 & 1.370 \\
\hline Pudiste evaluar programas educativos & PEE & 1.75 & 0.957 \\
\cline { 2 - 4 } & DOE & 3.67 & 1.496 \\
\cline { 2 - 4 } & MIDE & 2.10 & 1.524 \\
\hline Pudiste evaluar materiales y recursos & DOE & 2.75 & 0.957 \\
\cline { 2 - 4 } & MIDE & 1.91 & 1.612 \\
\cline { 2 - 4 } & PEE & 1.75 & 0.957 \\
\hline Pudiste evaluar instrumentos de recogida de datos & DOE & 2.50 & 1.713 \\
\cline { 2 - 4 } & MIDE & 2.36 & 1.362 \\
\hline & PEE & 2.25 & 1.500 \\
\hline & DOE & 2.44 & 1.504 \\
\cline { 2 - 4 } & MIDE & 2.18 & 1.401 \\
\hline
\end{tabular}

Tabla 3. Medias y desviaciones típicas de los objetivos referidos a habilidades en la dimensión consecución (cont.)

Los objetivos referidos a habilidades en cuanto a la dimensión consecución que obtienen medias bajas son, como se observa en la tabla 3, para el alumnado que es tutorizado por el profesorado del área DOE "pudiste diseñar programas educativos" (media 2.25 y desviación típica 1.48) y "pudiste evaluar programas educativos” (media 2.25 y desviación típica 1.61).

El alumnado que es tutorizado por el profesorado del área MIDE señala como el objetivo con media baja "pudiste diseñar instrumentos de recogida de datos" (media 1.82 y desviación típica 1.07).

Para el alumnado tutorizado por el profesorado del área PEE el objetivo "pudiste diseñar programas educativos” obtiene la media más baja (media 1.25 y desviación típica .50).

Finalmente, en la dimensión relacionada con la consecución de los objetivos a nivel actitudinal, como puede observarse en la tabla 4, el alumnado que es tutorizado tanto por el profesorado del área DOE y del área MIDE con medias altas es el objetivo "conseguiste demostrar una actitud positiva de cara a la investigación de los procesos involucrados en la práctica profesional del/de la psicopedagogo/a” con una media de 3.94 y desviación típica 1.06 para el primer grupo de alumnos/as y con una media 3.64 y una desviación típica de 1.36 para el otro grupo.

En cambio para el alumnado tutorizado por el profesorado del área PEE el objetivo con la media más alta es "conseguiste buscar alternativas prácticas que mejoren la 
calidad profesional del/de la psicopedagogo/a” con una media de 2.75 y desviación típica de .50 .

\begin{tabular}{|c|c|c|c|}
\hline \multirow{2}{*}{$\begin{array}{c}\text { Medias y desviaciones típicas de los objetivos actitudinales en la } \\
\text { dimensión consecución }\end{array}$} & \multicolumn{3}{|c|}{ Consecución } \\
\hline & Área & Media & DT \\
\hline \multirow{3}{*}{$\begin{array}{l}\text { Conseguiste demostrar una actitud positiva de cara a la investigación } \\
\text { de los procesos involucrados en la práctica profesional del } \\
\text { psicopedagogo/a }\end{array}$} & DOE & 3.94 & 1.063 \\
\hline & MIDE & 3.64 & 1.362 \\
\hline & PEE & 2.25 & 0.957 \\
\hline \multirow{3}{*}{$\begin{array}{l}\text { Conseguiste comprobar la efectividad de las técnicas a emplear por el } \\
\text { psicopedagogo/a }\end{array}$} & DOE & 3.56 & 1.365 \\
\hline & MIDE & 3.36 & 1.433 \\
\hline & PEE & 2.00 & 0.816 \\
\hline \multirow{3}{*}{$\begin{array}{l}\text { Conseguiste buscar alternativas prácticas que mejoren la calidad } \\
\text { profesional del psicopedagogo/a }\end{array}$} & DOE & 3.50 & 1.265 \\
\hline & MIDE & 3.27 & 1.489 \\
\hline & PEE & 2.75 & 0.500 \\
\hline
\end{tabular}

Tabla 4. Medias y desviaciones típicas de los objetivos actitudinales en la dimensión consecución

El objetivo que alcanza la media más baja para el alumnado que es tutorizado por el profesorado del área DOE y por el profesorado del área MIDE es "conseguiste buscar alternativas prácticas que mejoren la calidad profesional del/de la psicopedagogo/a” con una media de 3.50 y desviación típica 1.26 para los primeros y una media de 3.27 y desviación típica de 1.48 para los segundos (ver tabla 4).

Por el contrario, para el alumnado que es tutorizado por el profesorado del área PEE el objetivo con media más baja es "conseguiste comprobar la efectividad de las técnicas a emplear por el/la psicopedagogo/a” (media de 2 y desviación típica .81).

Si se realiza una valoración con respecto a la dimensión referida a la relevancia de los objetivos, atendiendo a los tres tipos de objetivos específicos (a nivel conceptual, de habilidades y de actitudes), se puede indicar que en cuanto a la dimensión relacionada con la relevancia de los objetivos a nivel conceptual, como puede apreciarse en la tabla 5, los que alcanzan la media más alta para el alumnado tutorizado por el profesorado del área DOE son "pudiste identificar problemas, aplicando los conocimientos teóricos-prácticos”(media 4.50 y desviación típica .73); “pudiste resolver problemas, aplicando los conocimientos teóricos-prácticos” (media 4.50 y desviación típica .63) y “pudiste identificar las funciones de la/el psicopedagoga/oa” (media 4.50 y desviación típica .63).

Para el alumnado que es tutorizado por el profesorado del área MIDE el que obtiene la media más alta es "pudiste identificar las funciones de la/el psicopedagoga/o" (media 4.64 y desviación típica .50).

El alumnado tutorizado por el profesorado del área PEE señala varios de los objetivos con una media de 5 y una desviación típica de 0: "pudiste identificar problemas, aplicando los conocimientos teórico-prácticos”; “pudiste resolver problemas, aplicando los conocimientos teóricos-prácticos” y "pudiste identificar programas encaminados a la solución y/o prevención de problemas”. 
Medias y desviaciones típicas de los objetivos conceptuales Dimensión Relevancia

\begin{tabular}{|c|c|c|c|}
\hline \multirow{2}{*}{ iviearas y uesviaciones upicas de ios ovjeuvos conceptuales } & \multicolumn{3}{|c|}{ Dimension reievancia } \\
\hline & Área & Media & DT \\
\hline \multirow{3}{*}{$\begin{array}{l}\text { Pudiste identificar problemas, aplicando los conocimientos teórico- } \\
\text { prácticos }\end{array}$} & DOE & 4.50 & .730 \\
\hline & MIDE & 4.27 & .905 \\
\hline & PEE & 5.00 & .000 \\
\hline \multirow[t]{3}{*}{ Pudiste resolver problemas, aplicando los conocimientos teórico-prácticos } & DOE & 4.50 & .632 \\
\hline & MIDE & 4.36 & .674 \\
\hline & PEE & 5.00 & .000 \\
\hline \multirow{3}{*}{$\begin{array}{l}\text { Pudiste identificar programas encaminados a la solución y/o prevención de } \\
\text { problemas }\end{array}$} & DOE & 4.19 & .750 \\
\hline & MIDE & 4.00 & .894 \\
\hline & PEE & 5.00 & .000 \\
\hline \multirow[t]{3}{*}{ Pudiste identificar las funciones de la/el psicopedagoga/o } & DOE & 4.50 & .632 \\
\hline & MIDE & 4.64 & .505 \\
\hline & PEE & 4.00 & 2.00 \\
\hline \multirow{3}{*}{$\begin{array}{l}\text { Pudiste evaluar críticamente las limitaciones de las funciones de la/el } \\
\text { psicopedagoga/o }\end{array}$} & DOE & 3.94 & .680 \\
\hline & MIDE & 4.27 & .647 \\
\hline & PEE & 3.75 & 1.893 \\
\hline
\end{tabular}

Tabla 5. Medias y desviaciones típicas de los objetivos conceptuales en la dimensión relevancia

Por el contrario, el objetivo que alcanza, ver la tabla 5, una media más baja para el alumnado tutorizado por el profesorado del área DOE es "pudiste evaluar críticamente las limitaciones de las funciones de la/el psicopedagoga/o" (media 3.94 y desviación típica .68).

Para el alumnado tutorizado por el profesorado del área MIDE es "pudiste identificar programas encaminados a la solución y/o prevención de problemas" (media 4 y deviación típica .89).

Y para el alumnado tutorizado por el profesorado del área PEE es "pudiste evaluar críticamente las limitaciones de las funciones de la/el psicopedagoga/o" (media 3.75 y desviación típica 1.89).

En la dimensión relacionada con la relevancia de los objetivos a nivel de habilidades, las medias más altas (ver en la tabla 6) para el alumnado tutorizado por el profesorado de DOE es "pudiste colaborar con personal especializado en la evaluación e intervención psicopedagógica” (media 4.13 y desviación típica 1.08).

Para el alumnado tutorizado por el profesorado de MIDE es "pudiste colaborar en el desarrollo de actividades pedagógicas” (media 4.82 y desviación típica .40).

Y, por último, para el alumnado tutorizado por el profesorado del área PEE son "pudiste conocer recursos disponibles en el medio institucional” y "pudiste diseñar instrumentos de recogida de datos" (ambos con una media de 4.75 y desviación típica de .50). 


\begin{tabular}{|c|c|c|c|}
\hline \multirow[t]{2}{*}{ Medias y desviaciones típicas de los objetivos referidos a habilidades } & \multicolumn{3}{|c|}{ Dimensión Relevancia } \\
\hline & Área & Media & DT \\
\hline \multirow{3}{*}{ Pudiste colaborar en la planificación de actividades pedagógicas } & DOE & 4.00 & .849 \\
\hline & MIDE & 4.64 & .674 \\
\hline & PEE & 3.75 & 1.893 \\
\hline \multirow[t]{3}{*}{ Pudiste colaborar en el desarrollo de actividades pedagógicas } & DOE & 4.06 & .680 \\
\hline & MIDE & 4.82 & .405 \\
\hline & PEE & 3.75 & 1.893 \\
\hline \multirow{3}{*}{$\begin{array}{l}\text { Pudiste colaborar con personal especializado en la evaluación e } \\
\text { intervención psicopedagógica }\end{array}$} & DOE & 4.13 & 1.088 \\
\hline & MIDE & 4.55 & .522 \\
\hline & PEE & 3.67 & 2.309 \\
\hline \multirow[t]{3}{*}{ Pudiste colaborar con otras/os profesionales en los procesos educativos } & DOE & 4.00 & .816 \\
\hline & MIDE & 4.64 & .505 \\
\hline & PEE & 3.75 & 1.893 \\
\hline \multirow[t]{3}{*}{ Pudiste conocer recursos disponibles en el medio institucional } & DOE & 3.69 & 1.014 \\
\hline & MIDE & 4.55 & .522 \\
\hline & PEE & 4.75 & .500 \\
\hline \multirow{3}{*}{ Pudiste adaptar recursos disponibles en el medio institucional } & DOE & 3.50 & .894 \\
\hline & MIDE & 4.55 & .522 \\
\hline & PEE & 4.15 & .500 \\
\hline \multirow[t]{3}{*}{ Pudiste diseñar programas educativos } & DOE & 3.13 & 1.258 \\
\hline & MIDE & 4.18 & .715 \\
\hline & PEE & 4.00 & 2.00 \\
\hline \multirow[t]{3}{*}{ Pudiste diseñar materiales y recursos necesarios } & DOE & 3.50 & 1.265 \\
\hline & MIDE & 4.09 & .701 \\
\hline & PEE & 4.50 & .577 \\
\hline \multirow[t]{3}{*}{ Pudiste diseñar instrumentos de recogida de datos } & DOE & 3.44 & 1.031 \\
\hline & MIDE & 4.09 & .701 \\
\hline & PEE & 4.75 & .500 \\
\hline \multirow[t]{3}{*}{ Pudiste aplicar programas educativos } & DOE & 3.56 & 1.365 \\
\hline & MIDE & 4.27 & .786 \\
\hline & PEE & 3.75 & 1.893 \\
\hline \multirow[t]{3}{*}{ Pudiste aplicar materiales y recursos } & DOE & 3.69 & 1.078 \\
\hline & MIDE & 4.18 & .751 \\
\hline & PEE & 4.67 & .577 \\
\hline \multirow[t]{3}{*}{ Pudiste aplicar instrumentos de recogida de datos } & DOE & 3.50 & 1.115 \\
\hline & MIDE & 4.09 & .701 \\
\hline & PEE & 4.50 & .577 \\
\hline \multirow[t]{3}{*}{ Pudiste sistematizar los datos recogidos (si los aplicaste) } & DOE & 3.67 & .976 \\
\hline & MIDE & 3.90 & .876 \\
\hline & PEE & 4.50 & .577 \\
\hline \multirow[t]{3}{*}{ Pudiste describir los datos recogidos (si los aplicaste) } & DOE & 3.60 & .986 \\
\hline & MIDE & 3.90 & .876 \\
\hline & PEE & 4.50 & .577 \\
\hline \multirow[t]{3}{*}{ Pudiste interpretar los datos recogidos (si los aplicaste) } & DOE & 3.53 & 1.187 \\
\hline & MIDE & 3.90 & .876 \\
\hline & PEE & 4.50 & .577 \\
\hline
\end{tabular}

Tabla 6. Medias y desviaciones típicas de los objetivos referidos a habilidades en la dimensión relevancia 


\begin{tabular}{llll} 
Medias y desviaciones típicas de los objetivos referidos a habilidades & \multicolumn{3}{c}{ Dimensión Relevancia } \\
\cline { 2 - 4 } & \multicolumn{1}{c}{ Área } & Media & \multicolumn{1}{c}{ DT } \\
\hline Pudiste evaluar programas educativos & DOE & 3.13 & 1.544 \\
\cline { 2 - 4 } & MIDE & 3.64 & .674 \\
\cline { 2 - 4 } & PEE & 4.50 & .577 \\
\hline Pudiste evaluar materiales y recursos & DOE & 3.50 & 1.155 \\
\cline { 2 - 4 } & MIDE & 3.64 & .674 \\
\hline Pudiste evaluar instrumentos de recogida de datos & PEE & 4.50 & .577 \\
\cline { 2 - 4 } & MOE & 3.19 & 1.109 \\
\cline { 2 - 4 } & MEE & 3.64 & .674 \\
\hline
\end{tabular}

Tabla 6. Medias y desviaciones típicas de los objetivos referidos a habilidades en la dimensión relevancia (cont.)

Por el contrario, en la tabla 6 también destacan los objetivos que alcanzan medias más bajas. Así para el alumnado tutorizado por el profesorado de DOE los objetivos son "pudiste diseñar programas educativos" (media 3.13 y desviación típica 1.25) y “pudiste evaluar programas educativos” (media 3.13 y desviación típica 1.54).

Para el alumnado tutorizado por el profesorado del área MIDE los objetivos con medias más bajas son "pudiste evaluar programas educativos"; "pudiste evaluar materiales y recursos" y "pudiste evaluar instrumentos de recogida de datos" (todos ellos con una media de 3.64 y desviación típica de .67).

Finalmente, para el alumnado que es tutorizado por el profesorado del área PEE el objetivo que obtiene una media más baja es "pudiste aplicar programas educativos" (media 3.75 y desviación típica 1.89).

Finalmente, en la dimensión relacionada con la relevancia de los objetivos a nivel actitudinal, todas las medias se sitúan por encima de 4 (ver tabla 7), siendo el objetivo mejor valorado, para el alumnado que es tutorizado por el profesorado del área DOE, el referido a "conseguiste comprobar la efectividad de las técnicas a emplear por el/la psicopedagogo/a” (media 4,31 y desviación típica 1.19), de acuerdo a lo reflejado en la tabla 7.

Para el alumnado que es tutorizado por el profesorado del área MIDE los objetivos son "conseguiste comprobar la efectividad de las técnicas a emplear por el/la psicopedagogo/a" y "conseguiste buscar alternativas prácticas que mejoren la calidad profesional del/de la psicopedagogo/a” (ambos con una media de 4.27 y desviación típica de 1.19).

Pero lo más destacado de este grupo de objetivos y que se observa en la tabla 7 es que el alumnado tutorizado por el profesorado del área PEE indica que los tres objetivos que se establecen a nivel actitudinal son muy importantes ya que sus medias son 4.50 y su desviación típica .57. 
Medias y desviaciones típicas de los objetivos actitudinales

\begin{tabular}{|c|c|c|c|}
\hline & \\
\hline & Área & Media & DT \\
\hline \multirow{3}{*}{$\begin{array}{l}\text { Conseguiste demostrar una actitud positiva de cara a la investigación de } \\
\text { los procesos involucrados en la práctica profesional del psicopedagogo/a }\end{array}$} & DOE & 4.25 & .775 \\
\hline & MIDE & 4.09 & 1.044 \\
\hline & PEE & 4.50 & .577 \\
\hline \multirow{3}{*}{$\begin{array}{l}\text { Conseguiste comprobar la efectividad de las técnicas a emplear por el } \\
\text { psicopedagogo/a }\end{array}$} & DOE & 4.31 & .793 \\
\hline & MIDE & 4.27 & 1.191 \\
\hline & PEE & 4.50 & .577 \\
\hline \multirow{3}{*}{$\begin{array}{l}\text { Conseguiste buscar alternativas prácticas que mejoren la calidad } \\
\text { profesional del psicopedagogo/a }\end{array}$} & DOE & 4.06 & .854 \\
\hline & MIDE & 4.27 & 1.191 \\
\hline & PEE & 4.50 & .577 \\
\hline
\end{tabular}

Tabla 7. Medias y desviaciones típicas de los objetivos actitudinales en la dimensión relevancia

Por el contrario, los objetivos que obtienen una media más baja, pero nunca inferior a 4, para el alumnado que es tutorizado por el profesorado del área DOE es “conseguiste buscar alternativas prácticas que mejoren la calidad profesional del/de la psicopedagogo/a” (media 4.06 y desviación típica .85) y para el alumnado tutorizado por el profesorado de MIDE es "conseguiste demostrar una actitud positiva de cara a la investigación de los procesos involucrados en la práctica profesional del/de la psicopedagogo/a” (media 4.09 y desviación típica 1.04).

Esta diferencia de medias observable entre las valoraciones realizadas por el alumnado sobre su percepción de los objetivos, en cuanto a las dimensiones de consecución y relevancia, fueron analizadas en una investigación previa (Espiñeira, Muñoz, Rebollo y Arias, 2013) a través de la prueba no paramétrica de Friedman para $\mathrm{K}$ muestras relacionadas a fin de establecer si eran estadísticamente significativas. Los resultados obtenidos nos permiten comprobar que, con un nivel de significación de $<.05$, la prueba Friedman fue estadísticamente significativa en 23 de los 26 ítems. Los tres ítems no significativos estadísticamente son "Pudiste colaborar en el desarrollo de actividades pedagógicas”, "Pudiste conocer recursos disponibles en el medio institucional” y "Pudiste aplicar materiales y recursos”, referidos a habilidades.

\begin{tabular}{|c|c|c|}
\hline & ÍTEMS & NIVEL DE SIGNIFICATIVIDAD \\
\hline \multirow{7}{*}{ 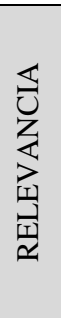 } & $\begin{array}{l}\text { Pudiste colaborar en el desarrollo de } \\
\text { pedagógicas }\end{array}$ & MIDE $>$ DOE $(p=.004)$ \\
\hline & \multirow{2}{*}{$\begin{array}{l}\text { Pudiste conocer recursos disponibles en el medio } \\
\text { institucional }\end{array}$} & MIDE > DOE $(p=.014)$ \\
\hline & & PEE > DOE $(p=.036)$ \\
\hline & \multirow{2}{*}{$\begin{array}{llllll}\begin{array}{l}\text { Pudiste adaptar } \\
\text { institucional }\end{array} & \text { recursos disponibles en } & \text { el medio } \\
\end{array}$} & MIDE > DOE $(\mathrm{p}=.003)$ \\
\hline & & PEE > DOE $(p=.018)$ \\
\hline & Pudiste diseñar instrumentos de recogida de datos & PEE $>$ DOE $(p=.011)$ \\
\hline & Pudiste evaluar instrumentos de recogida de datos & $\mathrm{PEE}>\mathrm{DOE}(\mathrm{p}=.022)$ \\
\hline \multirow{6}{*}{ 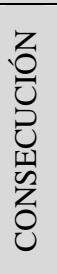 } & Pudiste describir los datos recogidos & DOE $>$ MIDE $(\mathrm{p}=.017)$ \\
\hline & $\begin{array}{l}\text { Pudiste identificar problemas , aplicando los conocimientos } \\
\text { teórico prácticos }\end{array}$ & DOE $>$ PEE $(p=.011)$ \\
\hline & \multirow[t]{2}{*}{ Pudiste identificar las funciones del /de la Psicopedagogo/a } & DOE $>$ PEE $(p=.016)$ \\
\hline & & MIDE > PEE $(p=.029)$ \\
\hline & \multirow[t]{2}{*}{ Pudiste interpretar los datos recogidos } & $\mathrm{DOE}>\mathrm{PEE}(\mathrm{p}=.035)$ \\
\hline & & DOE $>$ MIDE $(p=.019)$ \\
\hline
\end{tabular}

Tabla 8. Pruebas no paramétricas Kruskall Wallis y U-Manwhitney 
Para determinar si las diferencias observables son significativas, realizamos las pruebas no paramétricas Kruskall Wallis y U-Manwhitney, como se puede comprobar en la tabla 8. Así, se comprueba que de los 27 ítems que se analizan, 5 son estadísticamente significativos en la dimensión de relevancia y en la dimensión consecución sólo 4.

\section{Conclusiones}

En primer lugar, como conclusión general, podemos observar que el programa de Practicum presenta un buen desarrollo de la mayoría de los objetivos, sobre todo los referidos a habilidades, en donde las medias de los objetivos mejor valorados superan la puntuación de 4 . Se deberían potenciar más los objetivos que resultan ser más deficitarios y desarrollarlos más en los centros donde el alumnado realiza sus prácticas con metodologías adecuadas, lo que podría ser una nueva vía de investigación concretándose en la creación de programas específicos para potenciar un mayor desarrollo de éstos.

En segundo lugar, hemos de destacar la diferencia que se produce entre las puntuaciones referentes a la dimensión relevancia (más altas) con respecto a la dimensión consecución (más bajas). Ello parece hacernos concluir que el alumnado considera relevantes muchos de los objetivos a trabajar mediante el Practicum pero luego no son conseguidos con medias altas. No obstante, sí se suelen corresponder las puntuaciones de tal manera que los objetivos destacados como relevantes, son valorados positivamente con respecto a la consecución, como veremos a continuación.

Con respecto a los objetivos a nivel conceptual, hemos de señalar que los objetivos más relevantes, a juicio del alumnado, son los relacionados con la identificación y resolución de problemas, aplicación de los conocimientos teórico-prácticos, identificación de programas encaminados a la solución y/o prevención de problemas y finalmente, la identificación de funciones del/de la psicopedagogo/a. Estos objetivos, son señalados, igualmente por el alumnado en cuanto al nivel de consecución, excepto el relacionado con la resolución de problemas.

El objetivo relacionado con la evaluación crítica de las limitaciones de las funciones de la figura de psicopedagogo/a no es señalado por ningún/a alumno/a ni como el más relevante ni como el más conseguido.

Con respecto a los objetivos referidos a habilidades, hemos de señalar que los objetivos más relevantes, a juicio del alumnado, son los relacionados con el conocimiento de recursos durante el período de Practicum, con habilidades colaborativas (con personal especializado y en el desarrollo de actividades pedagógicas) y con el diseño de instrumentos de recogida de datos. En este caso, sólo el primero de los objetivos, referido al conocimiento de recursos y el relacionado con la colaboración en el desarrollo de actividades pedagógicas, es señalado por el alumnado. 
Los objetivos relacionados con el diseño, aplicación y evaluación de programas, instrumentos, materiales y recursos no son señalados por ningún/a alumno/a ni como los más relevantes ni como los más conseguidos.

Sólo MIDE y PEE consiguen alcanzar con puntuaciones altas los objetivos que mejor valoran en cuanto a la relevancia.

Con respecto a los objetivos actitudinales, hemos de señalar que todos los objetivos son relevantes, a juicio del alumnado. Sin embargo, el objetivo relacionado con comprobar la efectividad de las técnicas a emplear por el/la psicopedagogo/a no ha sido señalado por el alumnado como uno de los más conseguidos.

Sólo PEE consigue alcanzar con puntuaciones altas el objetivo que mejor valora en cuanto a la relevancia.

En tercer lugar, teniendo en cuenta los resultados obtenidos en cuanto a la dimensión consecución, en cuanto a los objetivos que han sido señalados como los más conseguidos, por áreas, siempre se han conseguido resultados más altos por parte del alumnado de DOE, MIDE en segundo lugar y PEE, en tercer lugar. Es por ello, que seguimos considerando interesante efectuar análisis relacionados con el área de conocimiento del profesorado tutor del alumnado del Practicum. Esta conclusión, va en la línea del estudio efectuado por Tello (2007), en el que se destacaban las materias de Diagnóstico en Educación, Modelos de Orientación y Orientación Profesional como aquellas de mayor utilidad para el desarrollo del Practicum.

La profundización en este tipo de análisis nos permitirá, en el futuro, ver si las diferencias se incrementan, se reducen o se mantienen. En función de ello, se podrían diseñar propuestas formativas en paralelo a los currícula formativos, o bien integradas en ellos, con el fin de conseguir profesionales competentes en estas temáticas.

Con respecto al análisis efectuado mediante las pruebas no paramétricas Kruskall Wallis y U-Manwhitney, y, teniendo en cuenta que la muestra es pequeña, no podemos afirmar rotundamente, las conclusiones extraídas, pero sí hemos detectado lo siguiente:

- Los ítems que muestran diferencias estadísticamente significativas, no son los mismos en la dimensión relevancia que en la dimensión consecución.

- Partíamos de la hipótesis de que algunos de los objetivos de la Licenciatura en Psicopedagogía serían superiores en el área MIDE y hemos comprobado que, con respecto a la dimensión consecución, el área significativa es DOE.

- Por otro lado, en la dimensión relevancia no hay un área que tenga diferencias significativas más altas sino que se reparten entre MIDE y PEE.

- En futuras investigaciones, y una vez ampliada la muestra, podremos estudiar si las diferencias detectadas se mantienen o no, así como realizar un análisis de resultados dependiendo del tipo de institución a la que haya acudido el alumnado. Este último matiz también había sido señalado en investigaciones anteriores (Tello, 2007). 


\section{Referencias bibliográficas}

ARMENGOL , C.; CASTRO , D.; JARIOT , M.; MASSOT , M. Y SALA , J. (eneroabril, 2011). El Practicum en el Espacio Europeo de Educación Superior: mapa de competencias del profesional de la educación. Revista de Educación, 354, 71-98.

BRETONES ROMÁN, A. (2013). El Practicum de magisterio en educación primaria: una mirada retrospectiva. Revista Complutense de Educación, 24(2), 443-471. Recuperado de http://revistas.ucm.es/index.php/RCED/article/view/42088

Cabrerizo Diago, J.; Rubio Roldán, M.J. y Castillo Arredondo, S. (2010). El Practicum en los grados de pedagogía, de magisterio y de educación social. Madrid: Pearson Educación.

CABRERIZO DIAGO, J.; RUBIO ROLDÁN, M.J., CASTILLO ARREDONDO, S. Y CABRERIZO GARCÍA, A. (2011). El Practicum del máster en formación del profesorado. Madrid: Pearson Educación.

CASTILLA MESA, M.T. (2009). El Practicum desde un modelo de formación por competencias. En Raposo, Rivas, M.; Martínez, Figueira, M.E.; Lodeiro, Enjo, L.; Fernández, de la Iglesia, J.C. y Pérez, Abellás, A. (Coords.). El Practicum más allá del empleo. Formación vs. Training (pp. 353-368). Pontevedra: Imprenta universitaria.

CID, A. Y OCAMPO, C.I. (septiembre-diciembre, 2007). Funciones tutoriales en el Practicum de Psicopedagogía en la Universidad de Vigo: percepción de los estudiantes actuales. Revista de Educación, 344, 285-307.

CUBO S. Y MONTANERO M. (2000). Propuesta de una estructura tridimensional para el diseño del Practicum de Psicopedagogía. Revista Española de Orientación y Psicopedagogía (REOP), 11(19), 121-137.

CUNILL MIR, F. (2009). Un modelo de coordinación y seguimiento de prácticas externas. En Raposo, Rivas, M.; Martínez, Figueira, M.E.; Lodeiro, Enjo, L.; Fernández, de la Iglesia, J.C. y Pérez, Abellás, A. (Coords.). El Practicum más allá del empleo. Formación vs. Training (pp. 407-418). Pontevedra: Imprenta universitaria.

DOMINGO ROGET, A. (2009). El Practicum en los nuevos planes de estudios. En Raposo, Rivas, M.; Martínez, Figueira, M.E.; Lodeiro, Enjo, L.; Fernández, de la Iglesia, J.C. y Pérez, Abellás, A. (Coords.). El Practicum más allá del empleo. Formación vs. Training (pp. 125-130). Pontevedra: Imprenta universitaria.

DOMINGO ROGET, A. (2009). El Practicum en los nuevos planes de estudios. En Raposo, Rivas, M.; Martínez, Figueira, M.E.; Lodeiro, Enjo, L.; Fernández, de la Iglesia, J.C. y Pérez, Abellás, A. (Coords.). El Practicum más allá del empleo. Formación vs. Training (pp. 407-418). Pontevedra: Imprenta universitaria.

ESPIÑEIRA, E.M.; MUÑOZ, J.M.; REBOLLO, N. Y ARIAS, M.A. (2013). El Practicum en la formación de psicopedagogos/as: análisis de las materias del área 
de métodos de investigación y diagnóstico en educación. En Centro de Investigaçao em Educaçao (CIEd) (pp. 2476-2489). Universidade do Minho.

GONZÁLEZ FERNÁNDEZ, N. (2006). Evaluación y mejora del Practicum en las titulaciones de Ciencias de la Educación de la UPV/EHU. Revista de Psicodidáctica, 11(1), 145-158.

LATORRE MEDINA, M.J. Y BLANCO ENCOMIENDA, F.J. (2009). El conocimiento de las percepciones y opiniones de los estudiantes y tutores de los centros externos sobre la incidencia del Practicum en la formación de los psicopedagogos. En Raposo, Rivas, M.; Martínez, Figueira, M.E.; Lodeiro, Enjo, L.; Fernández, de la Iglesia, J.C. y Pérez, Abellás, A. (Coords.). El Practicum más allá del empleo. Formación vs. Training (pp. 1279-1293). Pontevedra: Imprenta universitaria.

MARTÍN PÉREZ, A. (2008). Cómo mejorar el Practicum de Educación Social. Las Palmas de Gran Canaria: Universidad de las Palmas de Gran Canaria.

MARTíNEZ FIGUEIRA, M.E. (2005). La calidad del Practicum de psicopedagogía visto a través de los tutores de los centros de prácticas. En Raposo, M.; Sanmamed, M.; Iglesias, L.; Muradas, M. y Zabalza, M.A. (Coords.). El Practicum en el nuevo contexto del Espacio Europeo de Educación Superior (pp. 653-668). Pontevedra: Imprenta universitaria.

MARTÍNEZ FIGUEIRA, M.E. (2008). ¿Qué saben los tutores del Practicum de Psicopedagogía sobre la acción tutorial? Revista Española de Orientación y Psicopedagogía (REOP), 19(1), 73-77.

RODICIO, M.L. E IGLESIAS, M.J. (enero-abril, 2011). La formación en competencias a través del Practicum: un estudio piloto. Revista de Educación, 354, 99-124.

TELLO, J. (2007). Implicaciones formativas del Practicum de Psicopedagogía en los centros de educación secundaria. Profesorado. Revista de Currículum y Formación de Profesorado, 11(1), 1-16. Recuperado de http://www.ugr.es/local/recfpro/rev111COL2.pdf

VALLEJOS, A., FERNÁNDEZ, C. Y SÁNCHEZ E. (2002). El Practicum en Psicopedagogía en el C.E.S. Cardenal Espínola-CEU. Escuela Abierta, 5, 181-196.

ZABALZA, M.A. (2013). El Practicum y las prácticas en empresas. Madrid: Narcea. 


\section{Correspondencia con los autores}

Eva Ma ESPIÑEIRA BELLÓN

Alicia ARIAS RODRÍGUEZ

Nuria REBOLLO QUINTELA

Universidade da Coruña

Facultade de Ciencias da Educación

Departamento de Filosofía e Métodos de Investigación en Educación

Campus Elviña, s/n,

15071, A Coruña

e-mail: eva.espineira@udc.es 